\title{
Association of heart rate with cardiovascular events and mortality in hypertensive and normotensive population: a nationwide prospective cohort study
}

\author{
Yuebo Wang ${ }^{1,2 \#}$, Lu Yin ${ }^{1 \#}$, Bo Hu${ }^{1}$, Lap Ah Tse ${ }^{3}$, Yu Liu ${ }^{4}$, Haibin $\mathrm{Ma}^{5}$, Wei $\mathrm{Li}^{1}$; on behalf of PURE-China \\ Investigator group
}

${ }^{1}$ State Key Laboratory of Cardiovascular Disease, Fuwai Hospital, National Center for Cardiovascular Diseases, Peking Union Medical College and Chinese Academy of Medical Sciences, Beijing, China; ${ }^{2}$ Clinical Research Service Center, Henan Provincial People's Hospital, Zhengzhou University People's Hospital, Zhengzhou, China; ${ }^{3}$ Jockey Club School of Public Health and Primary Care, Faculty of Medicine, The Chinese University of Hong Kong, Hong Kong, China; ${ }^{4}$ Shenyang No. 242 Hospital, Shenyang, China; ${ }^{5}$ Xining Center for Disease Control and Prevention, Xining, China Contributions: (I) Conception and design: All authors; (II) Administrative support: W Li, L Yin; (III) Provision of study materials or patients: All authors; (IV) Collection and assembly of data: B Hu, H Ma, Y Liu; (V) Data analyses and interpretation: Y Wang, L Yin; (VI) Manuscript writing: All authors; (VII) Final approval of manuscript: All authors.

\#These authors contributed equally to this work.

Correspondence to: Prof. Wei Li. State Key Laboratory of Cardiovascular Disease, Fuwai Hospital, National Center for Cardiovascular Diseases, Peking Union Medical College and Chinese Academy of Medical Sciences, Fengcunxili, Mentougou District, Beijing 102300, China. Email: liwei@mrbc-nccd.com.

Background: Cardiovascular disease is the leading cause of death worldwide. We assessed the association of baseline heart rate with cardiovascular events and mortality in hypertensive and normotensive populations using a prospective urban and rural epidemiology cohort study in China.

Methods: A total of 29,554 individuals were involved in our analysis, distributed equally between groups of normotensive and hypertensive. The primary outcomes were myocardial infarction, stroke, major cardiovascular diseases, and cardiovascular mortality. Cox frailty models were utilized to estimate hazard ratios for cardiovascular outcomes, and restricted cubic splines were used to explore the shape of the association between baseline heart rate and cardiovascular mortality.

Results: During a total observational time of 230,813 person-years, 402 myocardial infarction events, 1,096 stroke events, 1,540 major cardiovascular events, and 356 cardiovascular deaths were documented. In adjusted analyses, normotensive subjects with baseline heart rate $>82.5$ beats per minute had a 3.30fold greater risk of cardiovascular death and an increased $72 \%$ risk of myocardial infarction, compared with individuals whose baseline heart rate was 65.5-71 beats per minute. A similar trend was observed for cardiovascular mortality in the hypertensive population, but the association was attenuated. Multivariableadjusted restricted cubic splines showed linear associations between baseline heart rate and cardiovascular mortality in two groups of people (all $\mathrm{P}<0.05$ for linearity).

Conclusions: Elevated baseline heart rate is associated with an increased risk of cardiovascular mortality and myocardial infarction in the normotensive population. The association is attenuated for cardiovascular death in hypertensive patients.

Keywords: Baseline heart rate; cardiovascular mortality; hypertensive; normotensive

Submitted Feb 11, 2021. Accepted for publication May 16, 2021.

doi: $10.21037 /$ atm-21-706

View this article at: http://dx.doi.org/10.21037/atm-21-706 


\section{Introduction}

Cardiovascular disease (CVD) is the leading cause of death worldwide, which has been known for many decades (1). And it was the top-ranked cause of disability-adjusted life years (DALYs) in 2019 according to the Global Burden of Disease Study by GBD Diseases and Injuries Collaborators (2). World Health Organization estimated that CVD would account for $>23$ million deaths by 2030 (3). Previous evidence suggested that heart rate is a well-known predictor of cardiovascular morbidity and mortality in normotensive and hypertensive populations $(4,5)$. As a result of a metaanalysis encompassing 848,320 subjects, 10 beats per min (bpm) increment of heart rate was associated with an increasing $8 \%$ risk of cardiovascular mortality (6). Notably, elevated heart rate has an impact on both peripheral and central blood pressures, and it is also related to an increased risk of developing hypertension $(7,8)$. However, it remains unclear whether present or absent hypertension could further influence heart rate-cardiovascular outcome associations.

Only 1 study compared the heart rate-mortality associations among hypertensive patients and the normotensive population, which found slightly elevated heart rate was associated with CVD death for hypertensive participants, but correlation abated for normotensive people (9). However, the influence of heart rate may be unrepresentative, due to the study conducted in 1 rural site of Henan Province, not at a national level. Other relevant studies only focused on normotensive or hypertensive populations (10-14), without comparison between them. Besides, the dose-response relations between heart rate and adverse outcomes in different research were inconsistent. The linear relations were observed in some studies $(6,15,16)$, in which the risk of cardiovascular mortality increased significantly with increasing heart rate. However, other studies indicated U-shaped curves between heart rate and mortality, suggesting excessively low heart rate was not beneficial (17-19).

Therefore, the primary objective of our study was to compare the associations between baseline heart rate and cardiovascular outcomes in normotensive populations and hypertensive patients, using a large-scale, multi-center cohort across 12 Chinese administrative regions. We also assessed the dose-response relations between baseline heart rate and the risk of cardiovascular mortality.

This article is presented following the STROBE reporting checklist (available at http://dx.doi.org/10.21037/ atm-21-706) (20).

\section{Methods}

\section{Study design and sample selection}

Our study data came from the Prospective Urban and Rural Epidemiology (PURE) study, which is a large, multicenter prospective cohort study that recruited 156,424 individuals in 17 low-income, middle-income, and highincome countries across 5 continents around the world. The design, participants selection, and methods of this global study have been published elsewhere previously $(21,22)$. China is 1 of the participating countries $(23,24)$. Briefly, 46,677 Chinese aged 35 to 70 years residing in 115 urban and rural communities across 12 provinces, municipalities, autonomous regions were enrolled from January 1 , 2005, to December 31, 2009, using a 1:1 rural-to-urban recruitment ratio. Multi-stage sampling was performed: (I) we chose administrative regions on maximizing economic and socio-cultural diversity purpose, which contained 3 socioeconomic regions, including four eastern regions (Beijing, Jiangsu, Shandong, and Liaoning), three central regions (Shanxi, Jiangxi, and Inner Mongolia), and five western regions (Yunnan, Qinghai, Shaanxi, Xinjiang, and Sichuan); (II) communities were sampled by urban and rural stratification; (III) all households in these communities were recruited if they had at least 1 eligible family member aged between 35-70 years; (IV) individuals aged between 35 and 70 years who intended to stay at the current address for the next 4 years were enrolled. Each participant signed written informed consent forms, before questionnaire interview, physical examination, and sample collection. The study was conducted in accordance with the Declaration of Helsinki (as revised in 2013). The protocol and informed consent were reviewed and approved by the institutional review board at Fuwai Hospital of Chinese Academy of Medical Sciences and Beijing Hypertension League Institute (No. 03-206).

\section{Data collection}

A standardized interview-administrated questionnaire was used to obtain information about demographic factors, socioeconomic status (education, location, region), lifestyles (smoking, alcohol intake, and physical activity), medical history (including medication use), and family history of CVD. Current smoking was defined as smoking at least 1 cigarette per day in the past 12 months. Current drinking was defined as drinking at least once per month in the past 12 months. Physical activity was assessed using the International Physical Activity Questionnaire, categorizing 
based on the metabolic equivalent of task (MET) per min per week into low (<600 MET-min per week), moderate (600-2,999 MET-min per week), and high ( $\geq 3,000$ METmin per week) (25). Family history of CVD was defined as the subject's parents or siblings were diagnosed with heart disease or stroke before.

Physical examination was conducted by trained physicians for each participant, including weight, height, peripheral blood pressure and heart rate. Subjects were asked to take the sitting position and placed their right arm on a table at heart level, using an Omron automatic digital blood pressure measuring device (Omron HEM-757; Omron, Kyoto, Japan) to record systolic blood pressure (SBP), diastolic blood pressure (DBP) and heart rate twice every 5-minute interval or longer. Before measurement, individuals were not allowed to smoke, exercise, eat, or climb stairs in 30 minutes or longer. Average peripheral blood pressure and heart rate were calculated from two repeated measurements. For the analysis of serum total cholesterol, the fasting blood sample of each participant was collected and measured in a centralized certified laboratory.

\section{Follow-ups}

The trained and dedicated research team contacted every participant at least every 1 year either by a face-to-face visit or by telephone using a standardized questionnaire to collect follow-up information. Event report forms, death certificates, and medical records were used to capture data about major cardiovascular events and death during followups, which were adjudicated centrally and regularly by a trained clinical event committee (CEC) using predefined definitions and International Classification of Diseases-10 (ICD-10) codes (26). Major cardiovascular events were defined as myocardial infarction (ICD-10 codes I21-I22), stroke (ICD-10 codes I60-I64, I69), heart failure (ICD-10 codes I50). Cardiovascular mortality was defined as death from myocardial infarction, stroke, heart failure, and other fatal cardiovascular causes such as fatal myocarditis (ICD10 codes I40) or arterial rupture of an aneurysm (ICD-10 codes I71-I72, I69). For the current analysis, we included all outcome events known in the PURE study database until March 31, 2017.

\section{Statistical analyses}

Continuous variables were presented as the mean and standard deviation (SD). Categorical variables were presented as numbers and corresponding percentages. We analyzed baseline heart rate as both a categorical and a continuous variable. As a categorical variable, baseline heart rate was classified into $<65.5,65.5-71$, $71-76,76-82.5, \geq 82.5 \mathrm{bpm}$ according to the quartiles and used the optimal range $(65.5-71 \mathrm{bpm})$ as the reference (4). As a continuous variable, $10 \mathrm{bpm}$ was used as a heart rate increase unit. The generalized linear model and Cochran-Mantel-Haenszel $\chi^{2}$ test were used to examine their mortality trend for continuous and categorical variables of baseline heart rate levels, respectively. Hazard ratios (HR) were calculated using multivariable Cox frailty analysis with random intercepts, accounting for the clustering of administrative regions (12 centers in China). In a minimally adjusted model, we adjusted for age, sex, smoking, drinking, Body Mass Index (BMI), family history of CVD, and center (as a random effect). In the second adjusted model, location (urban $v s$. rural), region (eastern, central, western), education, physical activity level, DBP and hypercholesterolemia were additionally added to adjust for. As sensitivity analyses, medication use (beta-blockers, calcium antagonist, angiotensinconverting enzyme inhibitors, angiotensin receptor blocker, and other types of antihypertensive drugs) was further adjusted for (Table S1). To create a score-matched cohort of individuals with hypertension and non-hypertension, a propensity score was calculated for each subject based on the baseline clinical variables (age, sex, BMI, and family history of CVD) from the logistic regressions. The nearest-neighborhood matching algorithm with caliper size specification $(0.25 \times$ standard deviation of propensity score) was used to perform a 1:1 matched analysis without replacement method using STATA 12.0 (STATA Corp., College Station, TX, USA). The balance of covariates was evaluated by estimating standardized mean difference (SMD) before and after matching, and the average absolute SMD $<0.1$ was considered successful balancing between the 2 groups (27). Restricted cubic splines with 4 knots and using the median as the reference category were utilized to explore the shape of the association between baseline heart rate and the outcomes (created by SAS LGTPHCURV9 Macro). Statistical analyses were performed with SAS 9.4 (SAS Institute Inc., Cary, NC). A P value less than 0.05 was considered statistically significant with a 2 -sided alternative.

\section{Results}

In this study, we excluded 1,538 subjects whose baseline 


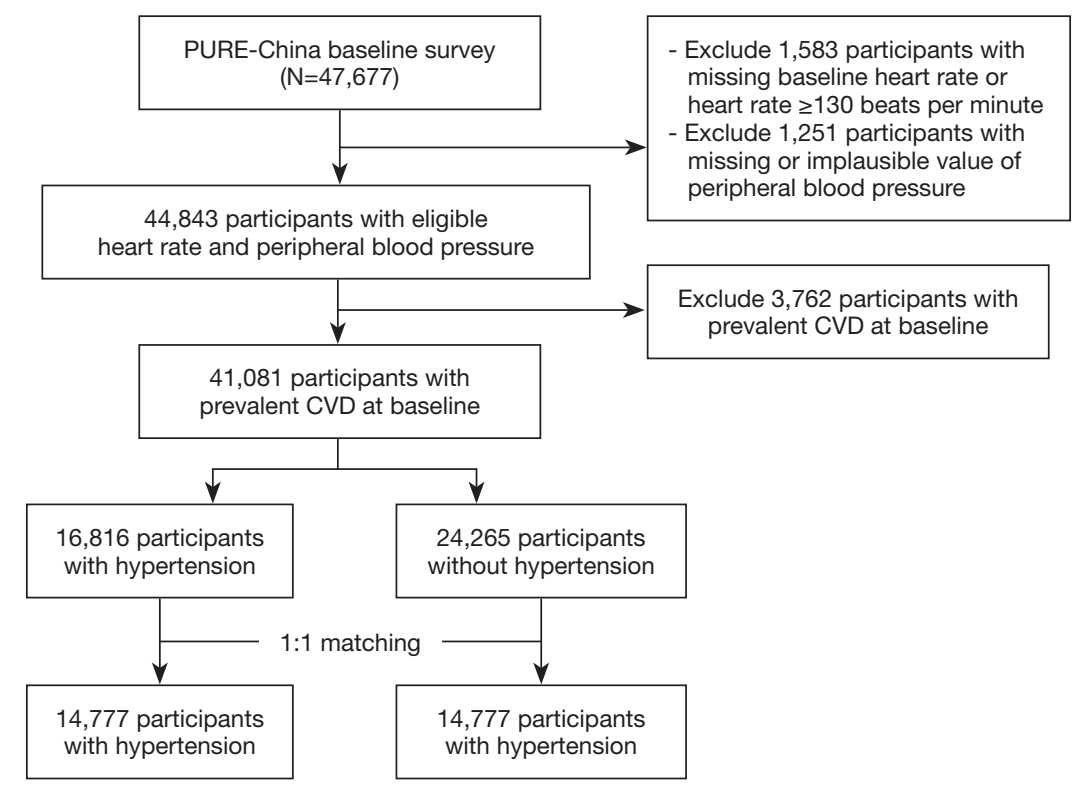

Figure 1 Flow chart of participant selection process. CVD, cardiovascular disease.

heart rates were missing or $\geq 130 \mathrm{bpm}$ at baseline, and 1,251 individuals without peripheral blood pressure measurement or with implausible values $(\mathrm{SBP}<70 \mathrm{mmHg}$ or SBP $>260 \mathrm{mmHg}$ or DBP $<40 \mathrm{mmHg}$ or DBP $>140 \mathrm{mmHg}$ ). Among 44,843 participants with complete baseline data, 3,762 individuals with prevalent CVD (angina/heart attack/coronary artery disease/heart failure/stroke and other heart diseases) recruited at baseline were deleted and remained of 16,816 hypertensive patients and 24,265 normotensive individuals in the datasets (hypertension defined as $\mathrm{SBP} \geq 140 \mathrm{mmHg}$ or $\mathrm{DBP} \geq 90 \mathrm{mmHg}$ or had diagnosed with hypertension by physicians or had antihypertensive medications). To balance differences in baseline characteristics between subjects with hypertension and normal blood pressure, a 1:1 propensity score-matched analysis was used to create the cohort. After matching, 14,777 individuals were included in each group for analysis. A detailed flow chart of participant selection was presented in Figure 1.

Table 1 showed the baseline characteristics of the hypertensive and normotensive participants stratified by heart rate categories. There were 14,777 participants in each hypertensive or normotensive group. The average baseline heart rate was $75.69 \pm 11.39 \mathrm{bpm}$ in the hypertension group, which was higher than that in the normotensive group $(73.23 \pm 9.96 \mathrm{bpm}, \mathrm{P}<0.001)$. After propensity score matching, the mean age $(53.12 \pm 9.30$ vs. $52.19 \pm 8.89$, in hypertension group $v s$. normotensive group order) and the average BMI (25.20 \pm 3.67 vs. 24.78 \pm 3.48$)$, sex composition (proportion of female: $55.8 \%$ vs. $55.9 \%$ ), the proportion of family history of CVD (21.5\% vs. $21.3 \%)$ were approximate between the two groups, and the average absolute SMD of them was 0.056 . In both groups, with the heart rate increase, DBP, prevalence of hypercholesterolemia, the proportion of low-level physical activity (<600 MET min per week) or female increased (all $\mathrm{P}_{\text {trend }}<0.05$ ). Whereas the proportion of current smokers or drinkers and individuals with a family history of CVD decreased (all $\mathrm{P}_{\text {trend }}<0.001$ ).

Table 2 described HRs and 95\% CIs of cardiovascular events and mortality with baseline heart rate in hypertensive and normotensive populations. During a total observational time of 230,813 person-years, 402 myocardial infarction events, 1,096 stroke events, 1,540 major cardiovascular events, and 356 cardiovascular deaths were documented. In hypertensive participants, cardiovascular mortality (HR, 1.13; 95\% CI, 1.02-1.25; $\mathrm{P}=0.017$ ) was associated with an increase of baseline heart rate by $10 \mathrm{bpm}$ in full-adjusted models. When baseline heart rate was categorized as five groups according to quartiles, defining $65.5-71 \mathrm{bpm}$ as the reference, hypertensive patients with baseline heart rate $<65.5 \mathrm{bpm}$ had 38\% (95\% CI, 0.40-0.96) decreased risk of cardiovascular mortality. However, myocardial infarction, stroke, and major cardiovascular events were not significantly associated with elevated baseline heart rate. 
Table 1 Baseline characteristics of hypertensive and normotensive participants according to quartiles of baseline heart rate

\begin{tabular}{|c|c|c|c|c|c|c|}
\hline Characteristics $^{\dagger}$ & \multicolumn{5}{|c|}{ Baseline heart rate categories (beats/minute) ${ }^{\ddagger}$} & $\mathrm{P}_{\text {trend }}{ }^{*}$ \\
\hline Hypertensive participants/n & 2,505 & 2,674 & 2,829 & 3,047 & 3,722 & \\
\hline Baseline heart rate (beats/minute) & $60.61 \pm 3.82$ & $68.11 \pm 1.60$ & $73.21 \pm 1.46$ & $78.78 \pm 1.86$ & $90.16 \pm 7.27$ & $<0.001$ \\
\hline Age (years) & $54.60 \pm 8.74$ & $53.68 \pm 9.05$ & $52.86 \pm 9.30$ & $52.45 \pm 9.42$ & $52.46 \pm 9.62$ & $<0.001$ \\
\hline Body mass index $\left(\mathrm{kg} / \mathrm{m}^{2}\right)$ & $25.01 \pm 3.53$ & $25.29 \pm 3.54$ & $25.28 \pm 3.67$ & $25.41 \pm 3.63$ & $25.02 \pm 3.86$ & 0.935 \\
\hline Family history of cardiovascular disease & $577(23.0)$ & $618(23.1)$ & $616(21.8)$ & $655(21.5)$ & 713 (19.2) & $<0.001$ \\
\hline Urban & $1,162(46.4)$ & $1,300(48.6)$ & $1,301(46.0)$ & $1,430(46.9)$ & $1,599(43.0)$ & $<0.001$ \\
\hline Region $^{\S}$ & & & & & & $<0.001$ \\
\hline Western & $492(19.6)$ & $561(21.0)$ & $585(20.7)$ & $664(21.8)$ & $942(25.3)$ & \\
\hline Education & & & & & & 0.678 \\
\hline Less than high school graduate & $973(38.9)$ & $998(37.4)$ & $1,076(38.1)$ & $1,130(37.2)$ & $1,458(39.4)$ & \\
\hline High school graduate & $1,364(54.6)$ & $1,465(55.0)$ & $1,536(54.4)$ & $1,684(55.5)$ & $1,999(54.0)$ & \\
\hline Some college or more & $162(6.5)$ & $202(7.6)$ & $212(7.5)$ & $222(7.3)$ & $248(6.7)$ & \\
\hline Currently a smoker & $702(28.0)$ & $656(24.5)$ & $648(22.9)$ & $640(21.0)$ & $783(21.0)$ & $<0.001$ \\
\hline Currently a drinker & $651(26.0)$ & $689(25.8)$ & $637(22.5)$ & $652(21.4)$ & $802(21.5)$ & $<0.001$ \\
\hline Physical activity level" (MET min per week) & & & & & & $<0.001$ \\
\hline Hypercholesterolemia & $199(7.9)$ & $263(9.8)$ & $254(9.0)$ & $289(9.5)$ & $413(11.1)$ & $<0.001$ \\
\hline Normotensive participants/n & 3,087 & 3,199 & 3,178 & 2,807 & 2,506 & \\
\hline Baseline heart rate (beats/minute) & $60.66 \pm 3.75$ & $68.11 \pm 1.59$ & $73.20 \pm 1.46$ & $78.71 \pm 1.84$ & $89.15 \pm 6.48$ & $<0.001$ \\
\hline Age (years) & $52.98 \pm 8.63$ & $52.19 \pm 8.72$ & $51.78 \pm 8.86$ & $51.72 \pm 9.17$ & $52.24 \pm 9.56$ & $<0.001$ \\
\hline Female sex & $1,379(44.7)$ & $1,731(54.1)$ & $1,881(59.2)$ & $1,703(60.7)$ & $1,572(62.7)$ & $<0.001$ \\
\hline Body mass index $\left(\mathrm{kg} / \mathrm{m}^{2}\right)$ & $24.58 \pm 3.26$ & $24.82 \pm 3.39$ & $24.95 \pm 3.39$ & $24.89 \pm 3.54$ & $24.62 \pm 3.85$ & 0.261 \\
\hline Family history of cardiovascular disease & $697(22.6)$ & $697(21.8)$ & $727(22.9)$ & $544(19.4)$ & $484(19.3)$ & $<0.001$ \\
\hline Urban & $1,551(50.2)$ & $1,688(52.8)$ & $1,734(54.6)$ & $1,490(53.1)$ & $1,216(48.5)$ & 0.471 \\
\hline Region & & & & & & 0.005 \\
\hline Eastern & $1,604(52.0)$ & $1,668(52.1)$ & $1,709(53.8)$ & $1,482(52.8)$ & $1,223(48.8)$ & \\
\hline Central & $766(24.8)$ & $794(24.8)$ & $722(22.7)$ & $640(22.8)$ & $601(24.0)$ & \\
\hline
\end{tabular}

Table 1 (continued) 
Table 1 (continued)

\begin{tabular}{|c|c|c|c|c|c|c|}
\hline Characteristics $^{\dagger}$ & \multicolumn{5}{|c|}{ Baseline heart rate categories (beats/minute) ${ }^{\ddagger}$} & $P_{\text {trend }}^{*}$ \\
\hline Western & $717(23.2)$ & $737(23.0)$ & $747(23.5)$ & $685(24.4)$ & $682(27.2)$ & \\
\hline Education & & & & & & 0.054 \\
\hline Less than high school graduate & $1,033(33.6)$ & $1,010(31.7)$ & $985(31.1)$ & $922(33.0)$ & $905(36.3)$ & \\
\hline Some college or more & $269(8.7)$ & $270(8.5)$ & $277(8.8)$ & $235(8.4)$ & $209(8.4)$ & \\
\hline Currently a smoker & $940(30.5)$ & $792(24.8)$ & $709(22.3)$ & $629(22.4)$ & $541(21.6)$ & $<0.001$ \\
\hline Currently a drinker & $827(26.8)$ & $714(22.3)$ & $639(20.1)$ & $528(18.8)$ & $467(18.6)$ & $<0.001$ \\
\hline Physical activity level (MET min per week) & & & & & & $<0.001$ \\
\hline$\geq 3,000$ & $1,401(46.3)$ & $1,314(41.7)$ & $1,380(43.9)$ & $1,162(42.0)$ & 988 (39.9) & \\
\hline Systolic blood pressure $(\mathrm{mmHg})$ & $121.10 \pm 10.87$ & $121.03 \pm 10.86$ & $120.89 \pm 10.77$ & $121.35 \pm 10.89$ & $121.43 \pm 11.06$ & 0.153 \\
\hline Diastolic blood pressure $(\mathrm{mmHg})$ & $74.59 \pm 7.60$ & $75.69 \pm 7.53$ & $76.51 \pm 7.19$ & $77.31 \pm 7.08$ & $78.23 \pm 7.07$ & $<0.001$ \\
\hline Hypercholesterolemia & $157(5.1)$ & $199(6.2)$ & $184(5.8)$ & $178(6.3)$ & $167(6.7)$ & 0.021 \\
\hline
\end{tabular}

${ }^{\dagger}$ Sum may not always add up to total because of missing values; ${ }^{\ddagger}$ Data are presented as $\mathrm{n}(\%)$ for categorial variables or mean \pm standard deviance for continuous variables; ${ }^{*} P_{\text {trend }}$ were obtained using generalized linear models for continuous variables and Mantel-Haenszel Chi-square trend test for categorical variables; ${ }^{\S}$ Eastern provinces (Beijing, Jiangsu, Shandong, and Liaoning), central provinces (Shanxi, Jiangxi, and Inner Mongolia), western provinces (Yunnan, Qinghai, Shaanxi, Xinjiang, and Sichuan); "Physical activity was assessed using the International Physical Activity Questionnaire, categorizing based on the metabolic equivalent of task (MET) per min per week into low (<600 MET min per week), moderate (600-3,000 MET min per week), and high ( $>3,000$ MET min per week). "Hypercholesterolemia defined as serum total cholesterol $>6.2 \mathrm{mmol} / \mathrm{L}$ or had diagnosed with hypercholesterolemia by physicians or had cholesterol-lowering medications.

The above associations for hypertensive subjects almost did not alter when further adjusted for use of heart rate lowering medications (beta-blockers, calcium antagonist) and antihypertension medications (angiotensin-converting enzyme inhibitors, angiotensin receptor blocker, and other types of antihypertensive drugs) (Table S1). A significant interaction between baseline heart rate and present or absent hypertension for cardiovascular mortality was observed ( $\mathrm{P}=0.016$ for interaction). Regarding normotensive participants, the magnitude of the association between cardiovascular deaths and baseline heart rate was enhanced. With an increase of baseline heart rate by $10 \mathrm{bpm}$, the risk increased $61 \%(95 \% \mathrm{CI}, 1.35-1.93 ; \mathrm{P} \leq 0.001)$. And normotensive subjects whose baseline heart rate $\geq 82.5 \mathrm{bpm}$ had a 3.30-fold (95\% CI, 1.72-6.32; $\left.\mathrm{P}_{\text {trend }}<0.001\right)$ greater risk than those with heart rate $65.5-71 \mathrm{bpm}$. Similar to the results of hypertension patients, the associations between baseline heart rate and stroke, major cardiovascular events were not significant among people with normal blood pressure. But when the baseline heart rate of normotensive subjects $\geq 82.5 \mathrm{bpm}$, the risk of developing myocardial infarction enhanced $72 \%$ (95\% CI, 1.02-2.90).

Figure 2 revealed the dose-response relations between baseline heart rate and the risk of study outcome. A significant linear relationship between baseline heart rate and cardiovascular mortality of normotensive participants ( $\mathrm{P}<0.001$ for linearity and $\mathrm{P}=0.144$ for non-linearity) was indicated by restricted cubic splines in fully adjusted model. In hypertensive patients, the association was moderately attenuated but still a linear relationship, the curve of which was flatter than that of normotensive subjects $(\mathrm{P}=0.019$ for linearity and $\mathrm{P}=0.766$ for non-linearity).

Table 3 showed adjusted HRs (95\% CIs) of cardiovascular mortality and myocardial infarction stratified by median 
Table 2 Association of baseline heart rate with cardiovascular events and mortality

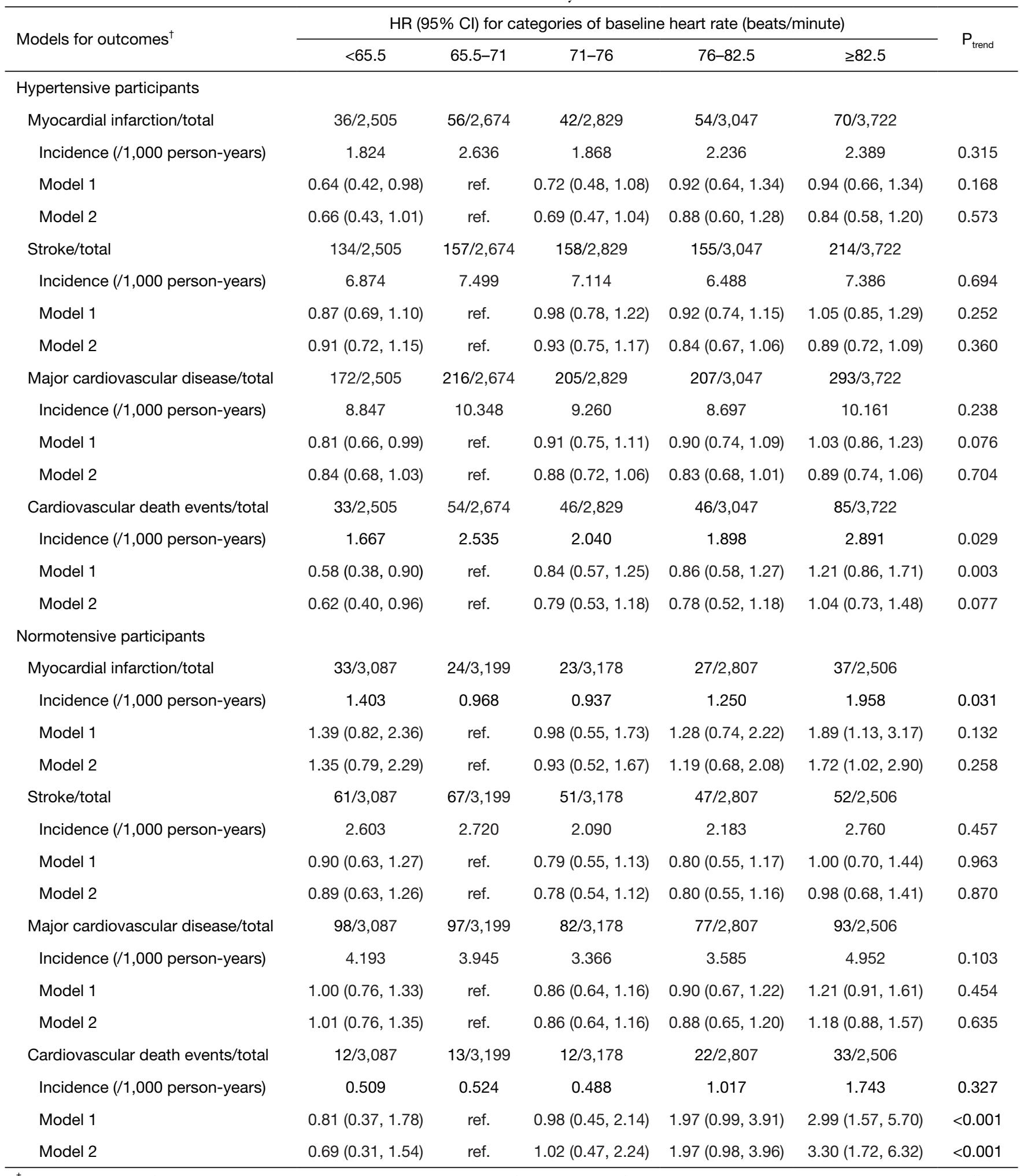

${ }^{\dagger}$ Model 1 adjusted for age, sex, smoking, drinking, body mass index, family history of cardiovascular disease and center as random effect; Model 2 adjusted for age, sex, smoking, drinking, body mass index, family history of cardiovascular disease, location, region, education, physical activity level, diastolic blood pressure, hypercholesterolemia and center as random effect. 

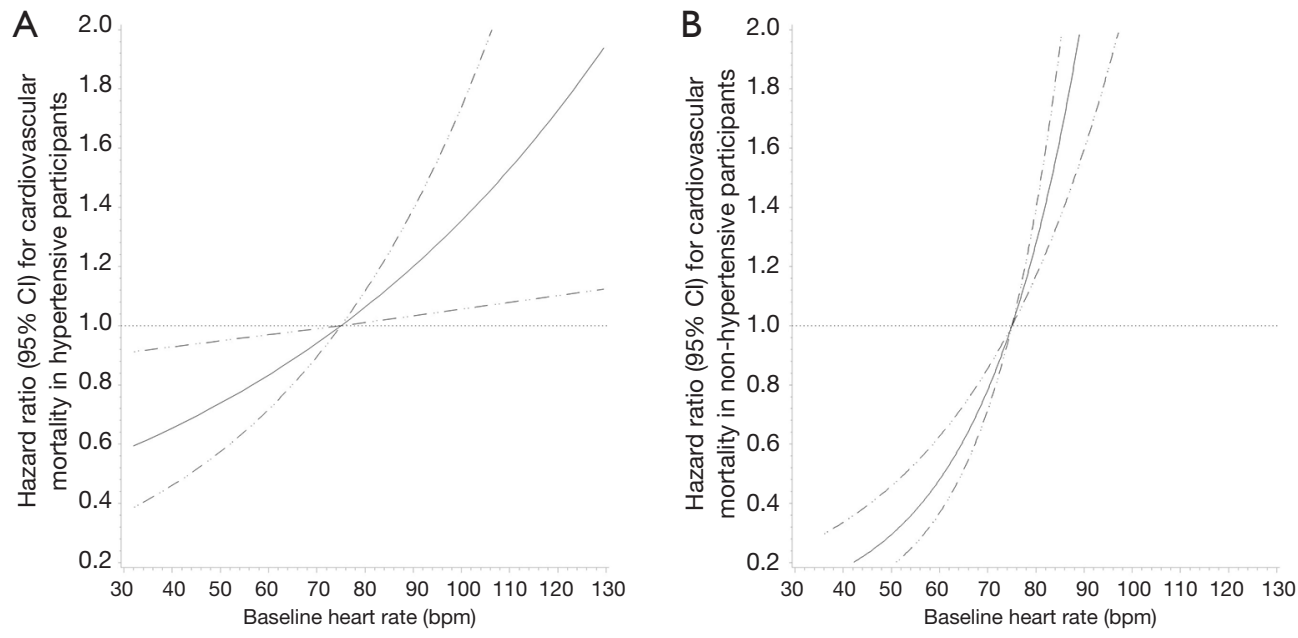

Figure 2 Restricted spline curve for association between baseline heart rate and the cardiovascular mortality in hypertensive participants (A) and normotensive participants (B). Adjusted for age, sex, smoking, drinking, Body Mass Index, family history of cardiovascular diseases, location, region, education, physical activity level, diastolic blood pressure, hypercholesterolemia. The solid line represented the value of hazard ratio; the dotted line represented the upper or lower limit of the $95 \%$ confidence interval for hazard ratio.

pulse pressure $(\mathrm{PP})$ of their respective population groups. The interactions were not observed between PP and baseline heart rate for cardiovascular mortality and myocardial infarction (all $\mathrm{P}>0.05$ for interaction). Among hypertension patients, compared with $\mathrm{PP}<58.5 \mathrm{mmHg}$ group, $\mathrm{PP} \geq 58.5 \mathrm{mmHg}$ group had a lower risk of cardiovascular mortality and myocardial infarction when their baseline heart rate decreased (heart rate $<65.5 \mathrm{bpm}$ group: $\mathrm{HR}_{\mathrm{CVD}-\text { mortality }}, 0.57$; $95 \% \mathrm{CI}, 0.35-0.91 ; \mathrm{HR}_{\mathrm{MI}}, 0.60$; 95\% CI, 0.37-0.98; heart rate 71-76 bpm group: $\mathrm{HR}_{\mathrm{MI}}$, $0.51 ; 95 \%$ CI, 0.30-0.87). As for normotensive people, the association between baseline heart rate and outcomes was greater significant in $\mathrm{PP} \geq 44.5 \mathrm{mmHg}$ group, when their heart rate was elevated (heart rate $\geq 82.5 \mathrm{bpm}$ group: $\mathrm{HR}_{\mathrm{CVD} \text {-mortality, }}$ 5.42; 95\% CI, 2.02-14.51; $\mathrm{HR}_{\mathrm{MI}}, 2.10 ; 95 \%$ CI, 1.07-4.14; heart rate 76-82.5 bpm group: $\mathrm{HR}_{\mathrm{CVD} \text {-mortality, }}$ $3.61 ; 95 \%$ CI, $1.29-10.06)$.

\section{Discussion}

In this large-scale population-based cohort study in China, we further confirmed the positive correlation between baseline heart rate and cardiovascular mortality, observing a significant interaction between baseline heart rate and present or absent hypertension. The magnitude of association between baseline heart rate and cardiovascular death in normotensive subjects was stronger than that in hypertensive people. Besides, baseline heart rate was also associated with myocardial infarction in normotensive people, especially for those whose heart rate above $82.5 \mathrm{bpm}$. The dose-response relationships between baseline heart rate and cardiovascular mortality were linear in these two groups of people. Furthermore, in the higher PP group, normotensive subjects with elevated baseline heart rate had markedly increased risk of cardiovascular death and myocardial infarction. As for hypertensive patients with higher PP, a lower baseline heart rate was associated with decreased risk of the above outcomes.

Consistent with the results of some studies $(6,28,29)$, our research revealed the positive effects of baseline heart rate on the risk of myocardial infarction and cardiovascular mortality in normotensive patients. The cut-off point of baseline heart rate was $82.5 \mathrm{bpm}$, close to the $80 \mathrm{bpm}$ in Tromso Study (28) or $84 \mathrm{bpm}$ in an Inner Mongolian cohort (29). In hypertension patients, there was a similar pattern for the risk of cardiovascular death, which was associated with an increase of heart rate by $10 \mathrm{bpm}$, but the magnitude was attenuated. When baseline heart rate was categorized into five groups, the association was not statistically significant (HR, 1.04; 95\% CI, 0.73-1.48) even in the highest group (heart rate $\geq 82.5 \mathrm{bpm}$ ). Meanwhile, we found a significant interaction between baseline heart rate and present or absent hypertension. The attenuated association in hypertensive patients could be due to the 
Table 3 Association of baseline heart rate with cardiovascular mortality and myocardial infarction by pulse pressure

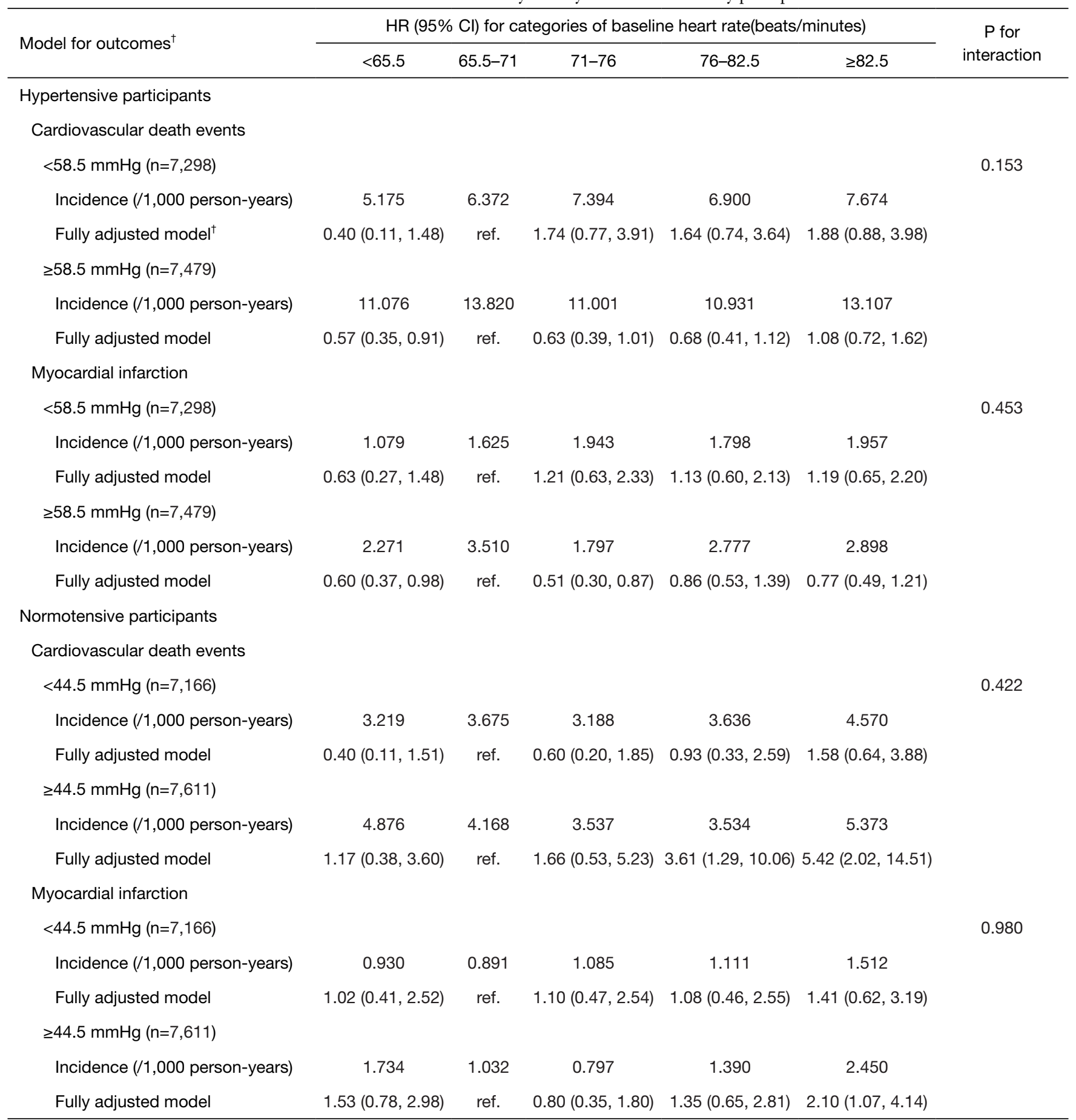

${ }^{\dagger}$ Model was adjusted for age, sex, smoking, drinking, body mass index, family history of cardiovascular diseases, location, region, education, physical activity level, hypercholesterolemia, and center as random effect. 
following reasons. Elevated heart rate is known to increase the peripheral blood pressure whereas it reduces the central blood pressure (4). Scott and Jamshed indicated that central aortic pressure may be a more significant clinical marker for increased cardiovascular risk than peripheral blood pressure $(4,8)$. A decrease in central blood pressure in hypertensive patients may contribute to better clinical outcomes. Therefore, we speculated that the effect of central blood pressure reduction mediated by elevated heart rate might play a more important role in hypertensive patients' outcomes. Further studies on pathological mechanisms are needed to explore their relationship. Besides, these results at least suggested that elevated baseline heart rate, as an early indicator of adverse cardiovascular outcomes, should be given equal attention in normotensive individuals.

Several previous prospective cohort studies have been reported the dose-response relation between baseline heart rate and cardiovascular morbidity or mortality. In the Gutenberg Health Study, a J-shaped mortality curve was illustrated in the general population, and each $10 \mathrm{bpm}$ baseline heart rate reduction was independently associated with increased mortality in heart rate $<64 \mathrm{bpm}$ subjects (30). Josep and his colleagues found that the HR continued a downslope in patients younger than 75 years old when their baseline heart rate $<60 \mathrm{bpm}$, but the HR revealed a J-shaped survival curve with a nadir at $68 \mathrm{bpm}$ in older patients (31). Our study revealed a significant linear relationship between baseline heart rate and cardiovascular mortality in both hypertensive and normotensive participants, and it was consistent with the result of a majority of research. For instance, Laura Paul observed that normotensive and untreated hypertensive individuals with decreasing heart rate had better outcomes during follow-up (32). And a meta-analysis including 10 studies revealed the protective effect of lower heart rate (45-69 bpm) on the risk of cardiovascular death (6). Different from studies conducted in hospitals, our subjects were recruited from the communities, which means they had fewer comorbidities and lower heart rate of participants is reflective of greater cardiorespiratory fitness (32), thus lower heart rate was linked to a greater survival rate. However, for hospitalized patients or elderly individuals, increased risk at lower baseline heart rate may be directly related to underlying worse clinical status or preexisting comorbidities, such as heart failure, diabetes (31). The optimal target heart rate of people with different characteristics is diverse, which still needs more research to be elucidated.

Among normotensive and hypertensive subjects, our study observed that elevated baseline heart rate and higher
PP had cumulative effects on cardiovascular mortality and myocardial infarction, but the interaction was not significant. It has been reported that as a component of cyclic stress, PP is linked with the stiffness of the aorta and other large arteries (33), and it might be more closely related to autonomic function than SBP or DBP (34). Previous studies documented that $\mathrm{PP}$ was also an independent risk marker for cardiovascular morbidity and mortality (35), and the combination of elevated heart rate and higher PP might have a synergistic effect on increasing the risk of cardiovascular events and mortality. Therefore, more health services should be provided to patients with high pp, especially for those whose heart rate increased simultaneously, which indicating a higher risk of adverse outcomes.

Our study had the same limitations as global PURE study $(22,36)$. Other limitations of this study should also be noted. Firstly, although our study indicates that a high baseline heart rate can be considered as a predictor of death from cardiovascular, the baseline heart rate might have changed over the long follow-up duration. Additionally, only two repeated heart rate measurements about fiveminute apart might be not enough to represent the actual heart rate. Secondly, as an observational study, we cannot completely adjust all potential confounders in particular for some unmeasured factors. Finally, China has breathtaking economic development and changes of the social factors behind, which resulted in unusual difficulties of follow-ups of PURE-China study, death events might be missed to report. Longer follow-up data should be used to evaluate the associations between heart rate and mortality, especially for time-varying heart rate.

\section{Conclusions}

In summary, our findings suggest that elevated baseline heart rate is associated with cardiovascular mortality and myocardial infarction in the Chinese normotensive population and the dose-response relationship is linear. The pattern of the association for cardiovascular mortality in hypertensive patients is similar to normotensive individuals', but the magnitude was attenuated. Thus, elevated baseline heart rate is supposed to be considered an early indicator of cardiovascular disease in both hypertensive and normotensive individuals. And heart rate monitoring should be given equal attention in people with normal blood pressure as it in hypertensive patients, to help health professionals to identify higher-risk individuals as early as possible. 


\section{Acknowledgments}

Besides co-authors listed in this study, we would like to thank those who supported our study and what they did with our sincere gratitude, especially for site coordinators, physicians, nurses, questionnaire interviewers, and laboratory personnel in all participating centers and communities.

Funding: This research was supported by the Population Health Research Institute, the Canadian Institutes of Health Research, Heart and Stroke Foundation of Ontario, and through unrestricted grants from several pharmaceutical companies. Besides, this work was also sponsored by CAMS Innovation Fund for Medical Sciences (CIFMS): 2016I2M-2-004, Construction of Basic Information Technology Support System and Platform for National Prevention and Treatment of Cardiovascular Diseases.

\section{Footnote}

Reporting Checklist: The authors have completed the STROBE (Strengthening the Reporting of Observational studies in Epidemiology) reporting checklist. Available at http://dx.doi.org/10.21037/atm-21-706

Data Sharing Statement: Available at http://dx.doi. org/10.21037/atm-21-706

Peer Review File: Available at http://dx.doi.org/10.21037/ atm-21-706

Conflicts of Interest: All authors have completed the ICMJE uniform disclosure form (available at http://dx.doi. org/10.21037/atm-21-706). The authors have no conflicts of interest to declare.

Ethical Statement: The authors are accountable for all aspects of the work and in ensuring that questions related to the accuracy or integrity of any part of the work are appropriately investigated and resolved. All procedures performed in this study involving human participants were in accordance with the Declaration of Helsinki (as revised in 2013). Each participant signed written informed consent forms, before questionnaire interview, physical examination and sample collection. The protocol and informed consent were reviewed and approved by the institutional review board at Fuwai Hospital of Chinese Academy of Medical Sciences and Beijing Hypertension League Institute (No.
$03-206)$

Open Access Statement: This is an Open Access article distributed in accordance with the Creative Commons Attribution-NonCommercial-NoDerivs 4.0 International License (CC BY-NC-ND 4.0), which permits the noncommercial replication and distribution of the article with the strict proviso that no changes or edits are made and the original work is properly cited (including links to both the formal publication through the relevant DOI and the license). See: https://creativecommons.org/licenses/by-nc-nd/4.0/.

\section{References}

1. Colantonio LD, Muntner P. It Is Time for Reducing Global Cardiovascular Mortality. Circulation 2019;140:726-8.

2. Collaborators GDaI. Global burden of 369 diseases and injuries in 204 countries and territories, 1990-2019: a systematic analysis for the Global Burden of Disease Study 2019. Lancet 2020;396:1204-22.

3. Organization WH. Projections of mortality and burden of disease, 2004-2030 [cited 2020 Jun 18] Available online: https://www.who.int/healthinfo/global_burden_disease/ projections2004 /en/.

4. Dalal J, Dasbiswas A, Sathyamurthy I, et al. Heart Rate in Hypertension: Review and Expert Opinion. Int J Hypertens 2019;2019:2087064.

5. Gillman MW, Kannel WB, Belanger A, et al. Influence of heart rate on mortality among persons with hypertension: the Framingham Study. Am Heart J 1993;125:1148-54.

6. Zhang D, Shen X, Qi X. Resting heart rate and all-cause and cardiovascular mortality in the general population: a meta-analysis. CMAJ 2016;188:E53-E63.

7. Kjeldsen SE. Hypertension and cardiovascular risk: General aspects. Pharmacol Res 2018;129:95-9.

8. Reule S, Drawz PE. Heart rate and blood pressure: any possible implications for management of hypertension? Curr Hypertens Rep 2012;14:478-84.

9. Zhang M, Han C, Wang C, et al. Association of resting heart rate and cardiovascular disease mortality in hypertensive and normotensive rural Chinese. J Cardiol 2017;69:779-84.

10. Alhalabi L, Singleton MJ, Oseni AO, et al. Relation of Higher Resting Heart Rate to Risk of Cardiovascular Versus Noncardiovascular Death. Am J Cardiol 2017;119:1003-7. 
11. Custodis F, Roggenbuck U, Lehmann N, et al. Resting heart rate is an independent predictor of all-cause mortality in the middle aged general population. Clin Res Cardiol 2016;105:601-12.

12. Jensen MT, Marott JL, Allin KH, et al. Resting heart rate is associated with cardiovascular and all-cause mortality after adjusting for inflammatory markers: the Copenhagen City Heart Study. Eur J Prev Cardiol 2012;19:102-8.

13. Jensen MT, Suadicani P, Hein HO, et al. Elevated resting heart rate, physical fitness and all-cause mortality: a 16year follow-up in the Copenhagen Male Study. Heart 2013;99:882-7.

14. Mao Q, Huang J-F, Lu X, et al. Heart rate influence on incidence of cardiovascular disease among adults in China. Int J Epidemiol 2010;39:1638-46.

15. Tverdal A, Hjellvik V, Selmer R. Heart rate and mortality from cardiovascular causes: a 12 year follow-up study of 379,843 men and women aged $40-45$ years. Eur Heart J 2008;29:2772-81.

16. Ho JE, Larson MG, Ghorbani A, et al. Long-term cardiovascular risks associated with an elevated heart rate: the Framingham Heart Study. J Am Heart Assoc 2014;3:e000668.

17. Hansen TW, Thijs L, Boggia J, et al. Prognostic value of ambulatory heart rate revisited in 6928 subjects from 6 populations. Hypertension 2008;52:229-35.

18. Nauman J, Nilsen TIL, Wisløff U, et al. Combined effect of resting heart rate and physical activity on ischaemic heart disease: mortality follow-up in a population study (the HUNT study, Norway). J Epidemiol Community Health 2010;64:175-81.

19. Woodward M, Webster R, Murakami Y, et al. The association between resting heart rate, cardiovascular disease and mortality: evidence from 112,680 men and women in 12 cohorts. Eur J Prev Cardiol 2014;21:719-26.

20. von Elm E, Altman DG, Egger M, et al. The Strengthening the Reporting of Observational Studies in Epidemiology (STROBE) statement: guidelines for reporting observational studies. Lancet 2007;370:1453-7.

21. Yusuf S, Rangarajan S, Teo K, et al. Cardiovascular risk and events in 17 low-, middle-, and high-income countries. N Engl J Med 2014;371:818-27.

22. Teo K, Chow CK, Vaz M, et al. The Prospective Urban Rural Epidemiology (PURE) study: examining the impact of societal influences on chronic noncommunicable diseases in low-, middle-, and high-income countries. Am Heart J 2009;158:1-7.e1.
23. Li W, Gu H, Teo KK, et al. Hypertension prevalence, awareness, treatment, and control in 115 rural and urban communities involving 47000 people from China. J Hypertens 2016;34:39-46.

24. Wang C, Hao G, Bo J, et al. Correlations between sleep patterns and cardiovascular diseases in a Chinese middleaged population. Chronobiol Int 2017;34:601-8.

25. Booth M. Assessment of physical activity: an international perspective. Res Q Exerc Sport 2000;71:S114-20.

26. Miller V, Mente A, Dehghan M, et al. Fruit, vegetable, and legume intake, and cardiovascular disease and deaths in 18 countries (PURE): a prospective cohort study. Lancet 2017;390:2037-49.

27. Stuart EA, Lee BK, Leacy FP. Prognostic score-based balance measures can be a useful diagnostic for propensity score methods in comparative effectiveness research. J Clin Epidemiol 2013;66:S84-S90.e1.

28. Sharashova E, Wilsgaard T, Mathiesen EB, et al. Resting heart rate predicts incident myocardial infarction, atrial fibrillation, ischaemic stroke and death in the general population: the Tromsø Study. J Epidemiol Community Health 2016;70:902-9.

29. Wang T, Zhang W, Zhang $M$, et al. Higher heart rates increase risk of diabetes and cardiovascular events: A prospective cohort study among Inner Mongolians. Diabetes Metab 2020;46:20-6.

30. Münzel T, Hahad O, Gori T, et al. Heart rate, mortality, and the relation with clinical and subclinical cardiovascular diseases: results from the Gutenberg Health Study. Clin Res Cardiol 2019;108:1313-23.

31. Lupón J, Domingo M, de Antonio M, et al. Aging and Heart Rate in Heart Failure: Clinical Implications for Long-term Mortality. Mayo Clin Proc 2015;90:765-72.

32. Paul L, Hastie CE, Li WS, et al. Resting heart rate pattern during follow-up and mortality in hypertensive patients. Hypertension 2010;5 5:567-74.

33. Yang X, Hidru TH, Han X, et al. Link Between Elevated Long-Term Resting Heart Rate Variability and Pulse Pressure Variability for All-Cause Mortality. J Am Heart Assoc 2020;9:e014122.

34. García-Ortiz L, Gómez-Marcos MA, Martín-Moreiras J, et al. Pulse pressure and nocturnal fall in blood pressure are predictors of vascular, cardiac and renal target organ damage in hypertensive patients (LOD-RISK study). Blood Press Monit 2009; 14:145-51.

35. Benetos A, Rudnichi A, Thomas F, et al. Influence of heart rate on mortality in a French population: role 
of age, gender, and blood pressure. Hypertension 1999;33:44-52.

36. Yin L, Deng G, Mente A, et al. Association patterns of urinary sodium, potassium, and their ratio with blood pressure across various levels of salt-diet regions in China. Sci Rep 2018;8:6727.

Cite this article as: Wang Y, Yin L, Hu B, Tse LA, Liu Y, Ma H, Li W; on behalf of PURE-China Investigator group. Association of heart rate with cardiovascular events and mortality in hypertensive and normotensive population: a nationwide prospective cohort study. Ann Transl Med 2021;9(11):917. doi: 10.21037/atm-21-706 
Supplementary

Table S1 Association of baseline heart rate with cardiovascular events and mortality in hypertensive participants (sensitivity analysis: further adjustment for confounding factors)

\begin{tabular}{|c|c|c|c|c|c|c|}
\hline Models for outcomes ${ }^{\dagger}$ & \multicolumn{5}{|c|}{$\mathrm{HR}(95 \% \mathrm{Cl})$ for categories of baseline heart rate (beats/minute) } & $P_{\text {trend }}$ \\
\hline \multicolumn{7}{|l|}{ Hypertensive participants } \\
\hline Myocardial infarction/Total & $36 / 2,505$ & $56 / 2,674$ & $42 / 2,829$ & $54 / 3,047$ & $70 / 3,722$ & \\
\hline Incidence (/1,000 person-years) & 1.824 & 2.636 & 1.868 & 2.236 & 2.389 & 0.315 \\
\hline Model 3 & $0.65(0.43,1.00)$ & ref. & $0.69(0.46,1.03)$ & $0.87(0.60,1.27)$ & $0.83(0.58,1.19)$ & 0.577 \\
\hline Model 4 & $0.65(0.43,0.99)$ & ref. & $0.69(0.46,1.03)$ & $0.87(0.60,1.27)$ & $0.83(0.58,1.19)$ & 0.557 \\
\hline Stroke/Total & $134 / 2,505$ & $157 / 2,674$ & $158 / 2,829$ & $155 / 3,047$ & $214 / 3,722$ & \\
\hline Incidence (/1,000 person-years) & 6.874 & 7.499 & 7.114 & 6.488 & 7.386 & 0.694 \\
\hline Model 4 & $0.91(0.72,1.15)$ & ref. & $0.93(0.75,1.17)$ & $0.84(0.67,1.05)$ & $0.88(0.72,1.09)$ & 0.342 \\
\hline Major cardiovascular disease /Total & $172 / 2,505$ & $216 / 2,674$ & $205 / 2,829$ & $207 / 3,047$ & $293 / 3,722$ & \\
\hline Incidence (/1,000 person-years) & 8.847 & 10.348 & 9.260 & 8.697 & 10.161 & 0.238 \\
\hline Model 2 & $0.84(0.68,1.03)$ & ref. & $0.88(0.72,1.06)$ & $0.83(0.68,1.01)$ & $0.89(0.74,1.06)$ & 0.704 \\
\hline Model 3 & $0.83(0.68,1.02)$ & ref. & $0.87(0.72,1.06)$ & $0.82(0.68,1.00)$ & $0.88(0.74,1.06)$ & 0.685 \\
\hline Model 4 & $0.84(0.68,1.02)$ & ref. & $0.88(0.72,1.06)$ & $0.83(0.68,1.00)$ & $0.88(0.74,1.06)$ & 0.683 \\
\hline Cardiovascular death events/Total & $33 / 2,505$ & $54 / 2,674$ & $46 / 2,829$ & $46 / 3,047$ & $85 / 3,722$ & \\
\hline Incidence (/1,000 person-years) & 1.667 & 2.535 & 2.040 & 1.898 & 2.891 & 0.029 \\
\hline
\end{tabular}

${ }^{\dagger}$ Model 2 adjusted for age, sex, smoking, drinking, Body Mass Index, family history of cardiovascular disease, location, region, education, physical activity level, diastolic blood pressure, Hypercholesterolemia and center as random effect; Model 3 adjusted for age, sex, smoking, drinking, Body Mass Index, family history of cardiovascular disease, location, region, education, physical activity level, diastolic blood pressure, Hypercholesterolemia, use of beta blocker, use of calcium antagonist and center as random effect; Model 4 adjusted for age, sex, smoking, drinking, Body Mass Index, family history of cardiovascular disease, location, region, education, physical activity level, diastolic blood pressure, Hypercholesterolemia, use of beta blocker, use of calcium antagonist, use of angiotensin converting enzyme inhibitors, use of angiotensin receptor blocker, use of other types of antihypertensive drugs and center as random effect. 